\title{
Retraction Note to: Improved fast handover method for multiple node by using mobile nodes guide
}

\author{
Radhwan M. Abdullah ${ }^{1}$. Zuriati Ahmad Zukarnain ${ }^{1}$
}

Published online: 7 March 2018

(c) Springer Science+Business Media, LLC, part of Springer Nature 2018

\section{Retraction Note to: \\ Telecommun Syst March 2017, Volume 64, Issue 3, pp 429-438$$
\text { https://doi.org/10.1007/s11235-016-0183-1 }
$$

The Editor-in-Chief has retracted this article [1] because it shows significant overlap with a previously published article [2]. The authors have not responded to any correspondence from the editor about this retraction.
[1] Abdullah, R.M., Zukarnain, Z.A. \& Iqbal, R. Telecommun Syst (2017) 64:429. https://doi.org/10.1007/s11235016-0183-1.

[2] Chiang, Meng-Shu et al. IET Networks (2015), 4(1):44. https://doi.org/10.1049/iet-net.2013.0150.

The original article can be found online at https://doi.org/10.1007/ s11235-016-0183-1.

$凶$ Zuriati Ahmad Zukarnain

Zuriati@upm.edu.my

Radhwan M. Abdullah

radwanmas@yahoo.com

1 Faculty of Computer Science and Information Technology,

University Putra Malaysia, UPM, 43400 Serdang, Selangor

D.E., Malaysia 